\title{
Hybrid and Bio Nanocomposites for Ultrasensitive Ammonia Sensors ${ }^{+}$
}

\author{
Jean-luc L. Wojkiewicz 1,*, Nathalie Redon ${ }^{1}$, Alexander Pud ${ }^{2}$, Sergei Mikhaylov ${ }^{2}$, \\ Nikolay Ogurtsov ${ }^{2}$, Yuriy Noskov ${ }^{2}$, Camille Collard ${ }^{1}$ and Wei Li ${ }^{1}$ \\ 1 SAGE-Département Sciences de l'Atmosphère et Génie de l'Environnement, IMT Lille Douai, \\ Université Lille, 59000 Lille, France; nathalie.redon@imt-lille-douai.fr (N.R.); \\ camille.collard1994@gmail.com (C.C.); wei.1.li@kcl.ac.uk (W.L.) \\ 2 Institute of Bioorganic Chemistry and Petrochemistry, National Academy of Science of Ukraine, \\ O21260 Kyiv, Ukraine; alexander.pud@gmail.com (A.P.); sergei.mikhaylov@mines-douai.fr (S.M.); \\ ogurtsov@bpci.kiev.ua (N.O.); noskov@bpci.kiev.ua (Y.N.) \\ * Correspondence: jean-luc.wojkiewicz@imt-lille-douai.fr; Tel.: +33-327-712-333 \\ † Presented at the Eurosensors 2017 Conference, Paris, France, 3-6 September 2017. \\ Published: 11 August 2017
}

\begin{abstract}
New organic electronic organic ammonia sensors based on hybrid nanocomposites polyaniline-titanium dioxide and on PANI/chitosan with high metrological performances were created. The physical and chemical properties of the nanocomposites were optimized to get a high sensitivity and very low detection threshold (in ppb values). For hybrids, in the core shell form, the performances were analyzed by the synergetic effects of the constituents. For biopolymers it is possible to get high performances ammonia sensors using the way of green chemistry. In both cases the nanocomposites worked at room temperature and showed linear responses with a response time around one minute.
\end{abstract}

Keywords: ammonia gas sensors; polyaniline; nanohybrid composites; biopolymers; chitosan

\section{Introduction}

Due to its intensive use, ammonia is becoming a major environmental pollutant. It is widely used in industry, agriculture (fertilizers) and comes from animal waste fermentation. In regards to its wide use, global ammonia emission in atmosphere increases continuously since the pre-industrial times promoting the formation of particulate matter and affecting flora, fauna and the quality of air $[1,2]$. The detection of ammonia at very low levels (ppm-ppb values) presents many interests for the control of ammonia concentration in atmosphere, for ultra-pure gases where ammonia can be a pollutant at ppb levels. Moreover the possibility to detect ammonia at these concentrations open new applications in medical diagnosis [3].

In regards to these environmental problems and its toxicity, the determination of ammonia concentration in atmosphere is subject of intensive research in the world. As classical chemical methods for determining ammonia concentration are time consuming, expensive and require trained personnel, electronic sensors were developed to get miniaturized devices with fast response and low cost. Among electronic gas sensors, it has been shown that conducting polymers and specifically "Polyaniline (PANI)" become a credible alternative to detect ammonia on a wide range of concentration and particularly at sub ppm levels where the other electronic sensors showed limitations $[4,5]$.

In this paper, two types of organic electronic ammonia sensors were studied in the ppm and sub ppm range. The first is based on a nano-hybrid composite $\mathrm{PANI}_{-\mathrm{TiO}_{2}}$ in the form of core-shells. The metrological performances were investigated showing the synergetic effects between the two materials. The second electronic sensor is based on a PANI/chitosan nanocomposite, showing that it 
is possible to use biopolymers and natural solvents to get efficient electronic sensors using the way of green chemistry.

\section{Materials and Measurements Methods}

\subsection{Materials}

PANI is a very promising material because it is easy to change its electronic properties not only by oxydo-reduction as other conducting polymers but also by acid-base reaction. This property is used to fabricate ammonia sensors. Emeraldine base is the insulating form of PANI. In the doping process an exchange of a proton from the acid dopant and the emeraldine base occurs giving the emeraldine salt, the conducting form of PANI. When PANI, in its conducting state, interacts with ammonia, there is an interaction with the proton on the emeraldine salt to create the ammonium ion $\mathrm{NH}_{4}{ }^{+}$, leading the localization of the polarons and an increase of the resistivity of the polymer as shown in the Figure 1. When the ammonia interaction stops the ammonium ion is decomposed to ammonia and a proton. The proton returns back to the polymer chain restoring the initial doping and the initial resistivity.

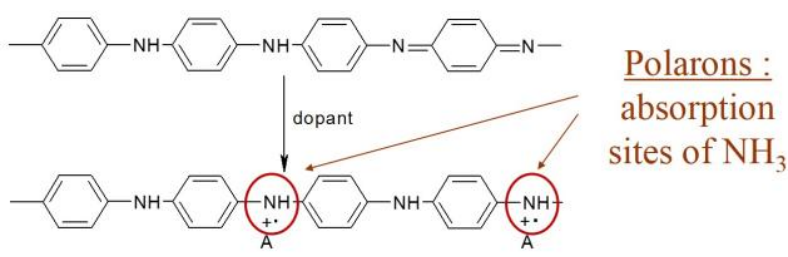

Figure 1. Polyaniline structure and ammonia interaction.

To enhance the sensor response to ammonia we studied hybrid nanomaterials $\mathrm{PANI}^{-\mathrm{TiO}_{2}}$ in the form of core shell structure. The core was constituted by titanium dioxide nanoparticles with a diameter of $10 \mathrm{~nm}$ and the shell by PANI doped with dodecyl sulfonic acid (DBSA) and lauryl sulfuric acid (LSA). The synthesis process and complete chemical and physical characterization has been already published [6]. The structure of the materials and the SEM image are shown in Figure 2.

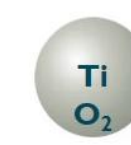

Bare nanoparticle

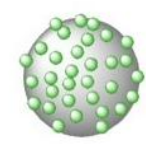

Monomer adsorption

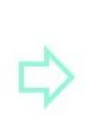

Core-Shell structure

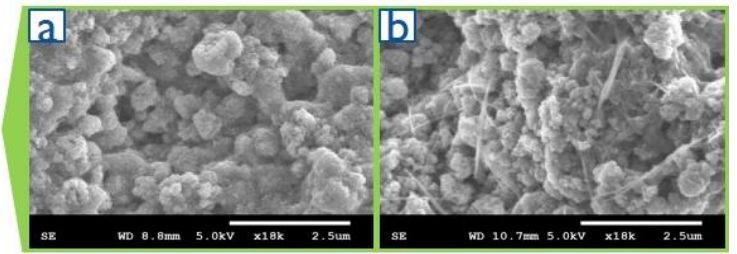

Figure 2. Core shell structure and SEM image of the materials ((a) PANI doped with DBSA; (b) PANI doped with LSA).

The second investigated way of materials synthesis and sensor fabrication is the use of natural and biodegradable material and natural acid for PANI dissolution. For that, we use Chitosan (D amino glucose) which comes from chitin (origin: crustacean; sea wastes) with a rate of deacetylation of $92.6 \%$. Chitosan is biocompatible and non-toxic (Figure 3). To elaborate the nanocomposites, the co-dissolution method was used. The polymer solution is deposited on interdigitated electrodes and dried for sensor fabrication
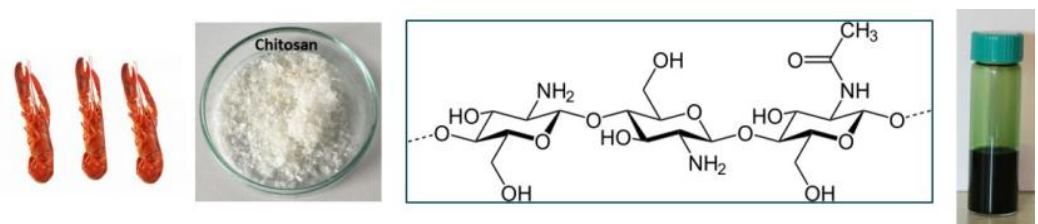

Figure 3. Chitosan and PANI-Chitosan solution. 
With this method a very low quantity of chemical products is used: with one liter of solution, it is possible to fabricate several millions of sensors. The cost of the sensing surface is negligible. The principle of measurement is based on the resistance variation of the sensing surface in regard to the gas concentration.

\subsection{Experimental Set up}

The sensors are deposited on an electronic card with interdigitated electrodes. Each card contains 100 sensors. As shown in Figure 4, four cards can be inserted in the exposure chamber for sensor characterization. That means that 400 sensors can be characterized is less than one minute. In the exposure chamber, gas concentration, temperature and relative humidity are controlled. The experiments are managed by Labview.
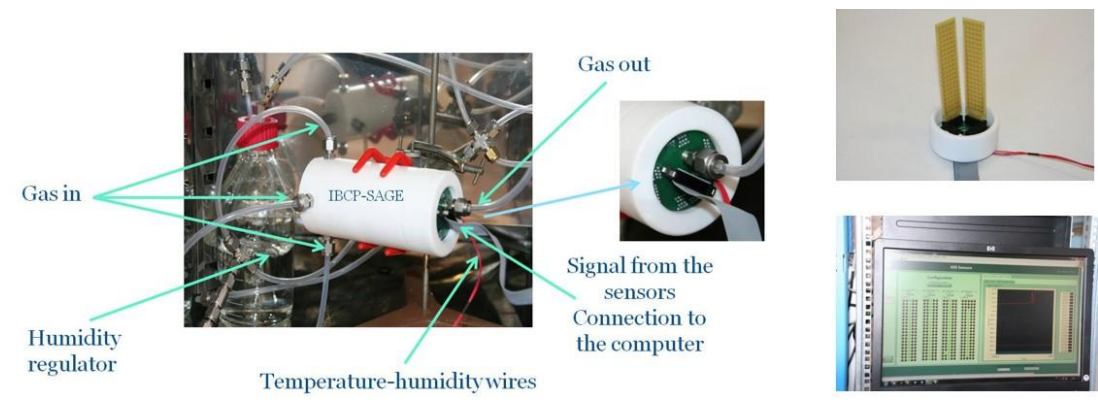

Figure 4. Exposure chamber and electronic cards.

\section{Results and Discussion}

\subsection{Hybrid PANI-TiO 2}

The typical response of the $\mathrm{PANI}-\mathrm{TiO}_{2}$ nanohybrids are shown in Figure 6a. it can be seen that the materials have a fast response and recovery time and a high sensitivity. We can observe that the response time increases when the ammonia concentration decreases. The quantification limit is estimated at $10 \mathrm{ppb}$. The Figure $6 \mathrm{~b}$ shows the comparison between pure PANI doped with DBSA and LSA and their corresponding hybrids ( $\mathrm{PANI}_{-T i O}$; $\mathrm{PANI}$ doped with the same acids). It can be seen that the hybrids form of materials have responses to ammonia 10 to 35 times greater than the pure form of PANI.
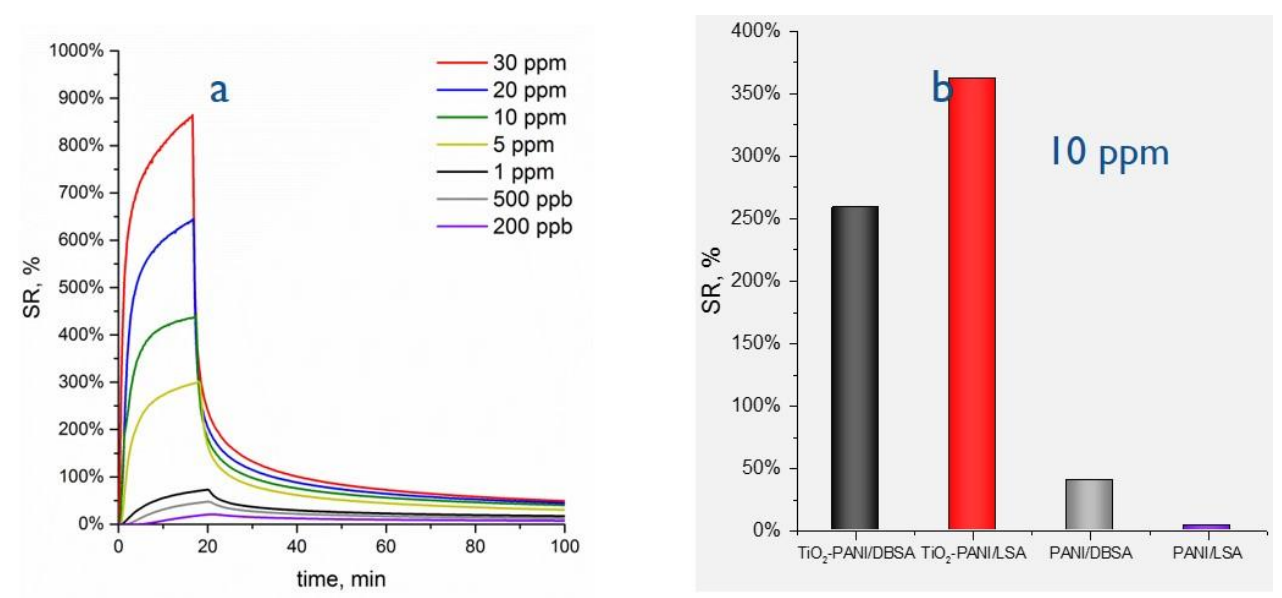

Figure 6. (a) Typical response of the hybrids to ammonia; (b) Comparison of the response of pure $\mathrm{PANI}$ and $\mathrm{PANI}-\mathrm{TiO}_{2}$ (doped with the same acids). 
The difference in the sensors respnse between pure PANI and $\mathrm{PANI}_{-} \mathrm{TiO}_{2}$ can be explained by the formation of a $\mathrm{P} \mathrm{N}$ heterojunction at the $\mathrm{PANI}^{-\mathrm{TiO}_{2}}$ nanoparticles interfaces. In these interfaces a depletion regions may appear, thus the conductivity is low due to the low local density of charges. When PANI is affected by ammonia interaction (deprotonation of PANI), the width of the depletion regions increases and the sensor signal is amplified.

\subsection{PANI-Chitosan Composites}

For PANI Chitosan, we get very stable solutions by strong hydrogen bonding between PANI and Chitosan [7]. The composites were studied in the sub ppm domain between 200 to $800 \mathrm{ppb}$. These first results showed response down to $200 \mathrm{ppb}$ of ammonia concentration with a linear variation of the response in regards to the ammonia concentration (Figure 7)
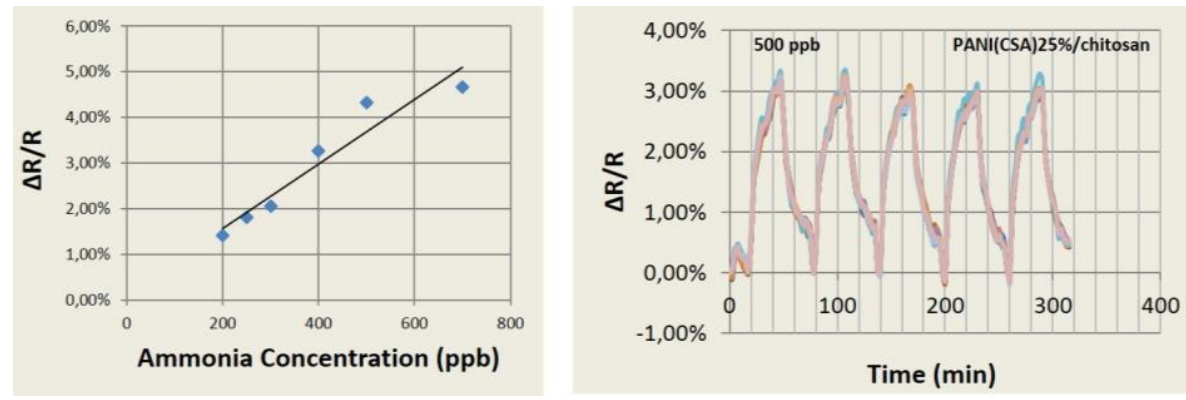

Figure 7. Response of PANI Chitosan composite to ammonia in the sub ppm range.

These senors showed a good reproducibility (10 different samples) and repeatability (5 cycles) demonstrating the possibility to get reliable electronic sensors for ammonia in the sub ppm range.

\section{Conclusions}

In this study, we have demonstrated that Polyaniline in the form of composites is an efficient material to fabricate electronic ammonia sensors with high metrological performances. These sensors are reversible, low cost, selective with low detection threshold and work on a wide range of pollutant concentration. The fabrication and the use do not need a great expense of energy and a totally green way is possible. They work at room temperature and can be produced at large scale. Nevertheless further investigations are needed to clarify all interaction mechanisms.

Conflicts of Interest: The authors declare no conflict of interest.

\section{References}

1. Krupa, S.V. Effects of atmospheric ammonia (NH3) on terrestrial vegetation: A review. Environ. Pollut. 2003, 124, 179-221.

2. Ruyet, J.P.-L.; Galland, R.; Le Roux, A.; Chartois, H. Chronic ammonia toxicity in juvenile turbot (Scophthalmus maximus). Aquaculture 1997, 154, 155-171.

3. Hibbard, T.; Crowley, K.; Killard, A.J. Direct measurement of ammonia in simulated breath using an inkjetprinted polyaniline nanoparticle sensor. Anal. Chem. Acta 2013, 779, 56-63.

4. Rizzo, G.; Arena, A.; Donato, N.; Latino, M.; Saitta, G.; Bonavita, A.; Neri, G. Flexible, all organic ammonia sensor based on dodecylbenzene sulfonic acid-doped polyaniline films. Thin Solid Films 2010, 518, 7133-7137.

5. Kebiche, H.; Debarnot, D.; Merzouki, A.; Poncin Epaillard, F.; Haddaoui, N. Relationship between ammonia sensing properties of polyaniline nanostructures and their deposition and synthesis methods. Anal. Chem. Acta 2012, 737, 64-71. 
6. Mikhaylov, S.; Ogurtsov, N.A.; Redon, N.; Coddeville, P.; Wojkiewicz, J.L.; Pud, A.A. The PANI-DBSA content and dispersing solvent as influencing parameters in sensing performances of $\mathrm{TiO}_{2} / \mathrm{PANI}^{-D B S A}$ hybrid nanocomposites to ammonia. RSC Adv. 2016, 6, 82625-82634.

7. Li, W.; Jang, D.M.; An, S.Y.; Kim, D.; Hong, S.K.; Kim, H. Polyaniline-chitosan nanocomposite: High performance hydrogen sensor from new principle. Sens. Actuators B Chem. 2011, 160, 1020-1025.

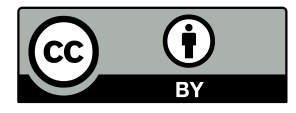

(C) 2017 by the authors. Licensee MDPI, Basel, Switzerland. This article is an open access article distributed under the terms and conditions of the Creative Commons Attribution (CC BY) license (http://creativecommons.org/licenses/by/4.0/). 